cure if it has advanced as far as this, still less if it involves the large trunks of the brachial plexus.

Having cleared the vessels, we may next proceed to detach the axillary contents from the lateral wall of the chest by quickly stripping up the thin fascia which attaches them to the serratus magnus and the ribs, avoiding injury to that muscle's nerve in doing so. The posterior wall is then to be cleaned, and this is frequently the most troublesome part of the procedure, on account of the hæmorrhage from the subscapular vessels. These should be exposed and caught before they are divided. The nerves, however, may nearly always be spared uninjured. The cutaneous nerves which cross the axilla may be sacrificed without much inconvenience. The whole mass, including the breast, contents of the axilla, and other istervening tissue, is to be removed entire by working outside it, so that it is not cut into. The external wound may be most quickly and easily closed by a continuous stitch of fine wire. If there is great tension I think the ordinary interrupted stitches serve best, starting from the angles towards the centre. They should be inserted near the edges and close together, about three or four to the inch, so as to distribute the tension equally. It is surprising how large a gap may safely be approximated in this way if the flaps have been well separated by the dissection. I have given up all kinds of button or deep stitches. I have not been able to give up the drainage-tube entirely. I drain the axilla by a tube perforating the posterior flap to carry away the blood and lymph, which are sometimes rather abundant and which may otherwise distend the flaps.

The proper putting up of a breast is important and not easy. Firm, smooth compression is required over a voluminous dressing. I use nothing but dry absorbent wool with a protection of gauze over the incision. As a rule two dreszing:s suffice-one in forty-eight hours to remove the tube, and the other on the ninth or tenth day to remove the stitches. The axillary portion invariably heals by the first intention, and the remainder also if care be taken in the putting up. Objections to this operation have been taken in the stiffness, cdema, numbness, and neuralgic pains which are said to follow it. In my experience inconvenience from these is not considerable and need not weigh in the slightest degree against the operation. Any cedema will be due to over-pressure in the axilla and soon passes away. Passive movements of the shoulder and elbow should be begun early and the movements of the arm become nearly perfect again with time and use.

The mortality from this operation is remarkably low. I do not think it should ever exceed 2 per cent. from causes proper to the operation. I have not had a death in seventysix consecutive cases, but one of my patients died from intestinal obstruction three weeks after operation. The old high death-rate was almost wholly due to septic diseases. I think shock is the principal danger to be feared, but by preventing loss of blood and unnecessary exposure to cold this is rarely formidable. The operation should be done as quickly as is consistent with thoroughness, and facility in doing it is to be acquired by practice. Occasionally some intercurrent disorder, such as bronchitis, may carry off a patient, but in every case the patient's fitness to bear the procedure must be considered on general surgical principles. There are a few other points to be noted. In planning the incisions regard must be had primarily for the efficient removal of the growth without reference to the subsequent approximation of the skin. But if the case is suitable for operation this can generally be accomplished. A small gap may be relied on to fill up quickly without suppuration if the wound be kept dry and aseptic. I have had some success in primary skin grafting, but it is uncertain. I have on occasions used a piece of the redundant skin which is cut away near the axilla. It is to be stripped of all fat and tissue and kept in warm boracic lotion till wanted. It may then be shaped to the gap, which it should nearly fill, and should be pressed down firmly so as to maintain close apposition of the raw surfaces. It is more certain of success, however, if the large piece is cut into small portions about a quarter of an inch square and planted near the edges. Operative grafting after eight or ten days is the most successful, a strip being taken from the inner side of the arm or thigh, but on many grounds it is objectionable. $I \mathrm{I}$ is useless to operate in cases of wide skin infiltration or with scattered tubercles, as the most extensive sacrifice of skin cannot cure these. Regionary recurrence in the skin and internal metastasis are to be expected.
If we find during an operation that the highest axillary gland is involved so that it is not possible to pass beyond the limits of the disease, what are we to do? I have no doubt, under these circumstances, the posterior triangle of the neck should be opened and cleared of its glands as an addition to the ordinary operation. There is a reasonable chance of getting beyond the limits of the disease. The incision may be prolonged upwards over the clavicle, but it is not necessary to divide or resect that bone, as has been done by some operators. I have on two occasions cleared the supra-clavicular fossa of its glands in this way, though, as they were rather advanced cases, the results were not very satisfactory, and the disease returned in osco.

\section{CASE OF SOLID FIBROID TUMOUR OF THE OVARY.}

BY C. E. PURSLOW, M.D., M.R.C.P. LOND.,

HONORARY OBSTHTRIC OFIICER, QUEEN'S HOSPITAL, BIRMINGHAM; ASSISTANT TO THE PROFESSORS OF MIDWITERY AND GYNECOLOGY, IIASON COLLEGL, BIRMINGHAM.

AN unmarried woman, aged twenty. six years, was admitted to the Queen's Hospital, Birmingham, on Dec. 10th, 1895, complaining of swelling of the abdomen. She had felt some pain and discomfort on the right side of the abdomen for five months, but the enlargement bad been noticed only a few days previously. Menstruation had been regular, but for the last six months the amount had been less than previously. She had had pain on defecation for four months, and for some weeks before admission had complained of frequent desire to micturate. On examination the abdomen was found to be considerably distended. A hard, nodular, irregular tumonr could be felt in the lower part of the abdomen reaching un to the umbilicus. The tumour moved freely under the band and appeared to float in some fluid, and gave a sensation of "ballottement" when pressure was made wish one hand, the other hand being applied to the opposite side of the abdomen. The physical sign obtained in this way resembled in a very marked degree that due to a foetus in utero. Bimanual examination, however, at once made it clear that the tumour was independent of the uterus. Percussion proved that the fluid in which the tumour floated was free in the prritoneal cavity. On Dec. 16th the abdomen was opened in the middle line. The incision was extended up to the umbilicus. About three pints of free ascitic fluid escaped. The tumour was then manipulated through the wound, and the pedicle. which proved to be a long one, was transfixed and tied with silk. The parietal peritoneum was seen to be injected, and its cut edge bled freely, but no adhesions had formed. The tumour grew from the right ovary and the left ovary was normal. No washing out or drainage was used, and the wound was closed by silkworm gut sutures. The wound healed by first intention. The patient made an uninterrupted recovery and left the hospital on the twenty-fourth day after the operation. The tumour, which was irregular and nodular in shape, weighed forty-one ounces; it was solid throughout. Sections of it were kindly examined under the microscope by Dr. Kauffman, patho. logist to the hospital, and he reported that it was composed entirely of fibroid tissue, which in some places was undergoing fatty degeneration.

All writers on gynæcology agree in stating that solid nonmalignant tumours of the ovary are rare, and in most of the text-books the subject is dismissed in a few lines. A full account of them is given in Pozzi's work on Gynæcology, and he mentions one or two characteristics which were present also in this case ; he states that they are almost invariably associated with the presence of free ascitic fluid and as a rule are free from adhesions. The Fallopian tube is usually quite free and is not adherent to the surface of the tumour, as is the case with cysts of the ovary; that is well seen in this specimen. He also states that they most often occur in comparatively young women. The presence of free ascitic fluid excites the suspicion of cancer, but in this case the fact that there was little pain and no loss of flesh was against that idea. For many of the notes and for valuable assistance in the treatment of the case $\mathrm{I}$ am indebted to $\mathrm{Mr}$. F. G. Messiter, obstetric house surgeon.

Birmingham. 